\title{
Timelike Surfaces With Constant Angle in de -Sitter Space $S_{1}^{3}$
}

\author{
Tuğba MERT ${ }^{1 *}$, Baki KARLIĞA² \\ ${ }^{I}$ Cumhuriyet University, Science Faculty, Sivas, Turkey \\ ${ }^{2}$ Gazi University, Science Faculty, Ankara, Turkey
}

Received: 21.01.2016; Accepted: 08.03.2016

\begin{abstract}
In this paper, we study a special class of timelike surface which is called constant timelike angle surfaces in de Sitter space $S_{1}^{3}$. In $S_{1}^{3}$, conditions being a constant angle timelike surface have been determined and invariants of these surface have been investigated. In here, we use the angle between unit normal vector field of surfaces and a fixed spacelike axis in ambient space.
\end{abstract}

Keywords: Constant angle surfaces , de Sitter space , Helix , Timelike surface

\section{De-Sitter Uzayında Sabit Açılı Zamansal Yüzeyler}

Özet. Bu çalışmada, yüzeyin birim normal vektör alanı ve $R_{1}^{4}$ de sabit bir uzaysal eksen arasındaki açıyı kullanarak, deSitter uzayında sabit zamansal açıl yüzeyler olarak adlandırılan zamansal yüzeylerin özel bir sınıfi geliştirilmiştir.

Anahtar Kelimeler: Sabit açılı yüzeyler, de-Sitter uzayı, Helis, zamansal yüzeyler

\section{INTRODUCTION}

In three dimensioan Euclidean space $E^{3}$, a constant angle surfaces are a surfaces whose tangents make constant angle with a fixed direction in ambient space. A surface whose tangent planes makes a constant angle with a fixed vector field is called constant angle surface in ambient space. M.I. Munteanu and A.I. Nistor studied constant angle surface and obtained all class of constant angle surface in $E^{3}$ [6]. Constant angle surface have been studied by A.J. Scale and G.R. Hernandez in ndimension Euclidean space $E^{n}[13,14]$. The Constant angle surface were applied to liquid layers and liquid crystals by P. Germelli and A.J. Scala [12]. Constant angle surface have been studied recently in product spaces $S^{2} \times R$ [15], $H^{2} \times R$ [16] or different ambient spaces $N i l_{3}$ [17]. In [1], Lopez and Munteanu studied constant hyperbolic angle surfaces whose unit normal timelike vector field makes a constant hyperbolic angle with a fixed timelike axis in Minkowski space $R_{1}^{4}$. In the literature constant timelike and spacelike angle surface have not been investigated both in hyperbolic space $H^{3}$ and de sitter space $S_{1}^{3}$. A constant timelike and a spacelike angle surface in Hyperbolic space $H^{3}$ and constant angle spacelike surface in de sitter space $S_{1}^{3}$ are developed in our paper [19], [20] and [21]. Constant timelike angle surface is a surface whose tangent planes makes a constant angle with a fixed vector field of $S_{1}^{3}$. De Sitter space is a good model for a physical phenomenon. This kind of surfaces in de Sitter space $S_{1}^{3}$ involved with our daily life such as architecture and geometrical design.

\footnotetext{
* Corresponding author. Email address: tmert@ cumhuriyet.edu.tr 


\section{MERT, KARLIĞA}

Probably, architectural structures and geometrical designs that use de Sitter curves enter into our life in the future. In this paper we introduce constant timelike angle timelike surfaces in de Sitter space $S_{1}^{3}$.

\section{PRELIMINIARIES}

Let $R_{1}^{4}$ be 4-dimensional vector space equipped with the scalar product $\langle$,$\rangle which is defined by$

$$
\langle x, y\rangle=-x_{1} y_{1}+x_{2} y_{2}+x_{3} y_{3}+x_{4} y_{4} \text {. }
$$

$R_{1}^{4}$ is 4-dimensional vector space equipped with the scalar product $\langle$,$\rangle , than R_{1}^{4}$ is called Lorentzian 4- space or 4-dimensional Minkowski space. The Lorentzian norm (length) of $x$ is defined to be

$$
\|x\|=|\langle x, x\rangle|^{\frac{1}{2}}
$$

If $\left(x_{0}^{i}, x_{1}^{i}, x_{2}^{i}, x_{3}^{i}\right)$ is the coordinate of $x_{i}$ with respect to canonical basis $\left\{e_{0}, e_{1}, e_{2}, e_{3}\right\}$ of $R_{1}^{4}$, then the lorentzian cross product $x_{1} \times x_{2} \times x_{3}$ is defined by the symbolic determinant

$$
x_{1} \times x_{2} \times x_{3}=\left|\begin{array}{cccc}
-e_{0} & e_{1} & e_{2} & e_{3} \\
x_{0}^{1} & x_{1}^{1} & x_{2}^{1} & x_{3}^{1} \\
x_{0}^{2} & x_{1}^{2} & x_{2}^{2} & x_{3}^{2} \\
x_{0}^{3} & x_{1}^{3} & x_{2}^{3} & x_{3}^{3}
\end{array}\right| \text {. }
$$

On can easly see that

$$
\left\langle x_{1} \times x_{2} \times x_{3}, x_{4}\right\rangle=\operatorname{det}\left(x_{1}, x_{2}, x_{3}, x_{4}\right) .
$$

In [2],[3]and [5] Izimuya at all introduced and investigated differantial geometry of curves and surfaces in Hyperbolic 3-space. In the rest of this section, we give background of context in [22].

Given a vector $v \in R_{1}^{4}$ and a real number $c$, the hyperplane with pseudo normal $v$ is defined by

$$
H P(v, c)=\left\{x \in R_{1}^{4},\langle x, v\rangle=c\right\} .
$$

We say that $H P(v, c)$ is a spacelike hyperplane, timelike hyperplane or lightlike hyperplane if $v$ is timelike, spacelike or lightlike respectively. We have following three types of pseudo-spheres in $R_{1}^{4}$ :

$$
\begin{aligned}
& \text { Hyperbolic-3 space : } H^{3}(-1)=\left\{x \in R_{1}^{4},\langle x, x\rangle=-1, x_{0} \geq 1\right\} \text {, } \\
& \text { de Sitter 3- space : } S_{1}^{3}=\left\{x \in R_{1}^{4},\langle x, x\rangle=1\right\}, \\
& \text { (open) lightcone : } L C^{*}=\left\{x \in R_{1}^{4} /\{0\},\langle x, x\rangle=0, x_{0}=1\right\} .
\end{aligned}
$$

We also define the lightcone 3 -sphere

$$
S_{+}^{3}=\left\{x \in R_{1}^{4},\langle x, x\rangle=0, x_{0}=1\right\} .
$$


A hypersurface given by the intersection of $S_{1}^{3}$ with a spacelike (resp.timelike) hyperplane is called an elliptic hyperquadric (resp. hyperbolic hyperquadric). If $c \neq 0$ and $\operatorname{HP}(v, c)$ is lightlike, then $H P(v, c) \cap S_{1}^{3}$ is a de Sitter horosphere.

Let $U \subset I R^{2}$ be open subset, and let $x: U \rightarrow S_{1}^{3}$ be an embedding. If the vector subspace $\tilde{U}$ which generated by $\left\{x_{u_{1}}, x_{u_{2}}\right\}$ is spacelike, then $x$ is called spacelike surface, if $U$ contain at least a timelike vector field then $x$ is called timelike surface in $S_{1}^{3}$.

In point of view Kasedou [22], we construct the extrinsic differantial geometry of curves in $S_{1}^{3}$. Since $S_{1}^{3}$ is a Riemannian manifold, the regular curve $\gamma: I \rightarrow S_{1}^{3}$ is given by arclength parameter.

\section{Theorem 1}

i) if $\gamma: I \rightarrow S_{1}^{3}$ is a spacelike curve with unit speed, then Frenet-Serre type formulae is obtained

$$
\left\{\begin{array}{l}
\gamma^{\prime}(s)=t(s) \\
t^{\prime}(s)=\kappa_{d}(s) n(s)-\gamma(s) \\
n^{\prime}(s)=-\kappa_{d}(s) t(s)-\tau_{d}(s) e(s) \\
e^{\prime}(s)=-\tau_{d}(s) n(s)
\end{array}\right.
$$

where $\kappa_{d}(s)=\left\|t^{\prime}(s)+\gamma(s)\right\|$ and $\tau_{d}(s)=-\frac{\operatorname{det}\left(\gamma(s), \gamma^{\prime}(s), \gamma^{\prime \prime}(s), \gamma^{\prime \prime \prime}(s)\right)}{\kappa_{d}^{2}(s)}$.

ii) If $\gamma: I \rightarrow S_{1}^{3}$ is a timelike curve with unit speed, then Frenet-Serre type formulae is obtained

$$
\left\{\begin{array}{l}
\gamma^{\prime}(s)=t(s) \\
t^{\prime}(s)=\kappa_{d}(s) n(s)+\gamma(s) \\
n^{\prime}(s)=\kappa_{d}(s) t(s)+\tau_{d}(s) e(s) \\
e^{\prime}(s)=-\tau_{d}(s) n(s)
\end{array}\right.
$$

where $\kappa_{d}(s)=\left\|t^{\prime}(s)-\gamma(s)\right\|$ and $\tau_{d}(s)=-\frac{\operatorname{det}\left(\gamma(s), \gamma^{\prime}(s), \gamma^{\prime \prime}(s), \gamma^{\prime \prime \prime}(s)\right)}{\kappa_{d}^{2}(s)}$.

It is easily see that $\kappa_{d}(s)=0$ if and only if there exists a lightlike vector $c$ such that $\gamma(s)-c$ is a geodesic.

Now we give extrinsic differential geometry on surfaces in $S_{1}^{3}$ due to Kasedou [22].

Let $U \subset I R^{2}$ is an open subset, and $x: U \rightarrow S_{1}^{3}$ is a regular surface $M=x(u)$. Since $M$ is a timelike surface, there is $\mathrm{e}\left(e(u)=\frac{x(u) \wedge x_{1}(u) \wedge x_{2}(u)}{\left\|x(u) \wedge x_{1}(u) \wedge x_{2}(u)\right\|}\right.$ such that $\langle e, x\rangle=\left\langle e, x_{u_{i}}\right\rangle=0,\langle e, e\rangle=1$. Thus there is de Sitter Gauss image of $x$ which is defined by mapping $E: U \subset I R^{2} \rightarrow S_{1}^{3}, E(u)=e(u)$. The lightcone Gauss image of $x$ is defined by map $L^{ \pm}: U \rightarrow L C^{*}$

$$
L^{ \pm}(u)=x(u) \pm e(u)
$$

The derivative $d x\left(u_{0}\right)$ can be identify by the mapping $1_{T_{p} M}$ on the tangent space $T_{p} M$. 


\section{MERT, KARLIĞA}

Therefore, we have

$$
d L^{ \pm}\left(u_{0}\right)=1_{T_{P} M} \pm d E\left(u_{0}\right)
$$

The linear transformations

$$
S_{p}^{ \pm}:=-d L^{ \pm}\left(u_{0}\right): T_{p} M \rightarrow T_{p} M
$$

and

$$
A_{p}:=-d E\left(u_{0}\right): T_{p} M \rightarrow T_{p} M
$$

is called the hyperbolic shape operator and de Sitter shape operator of $M$ at $p=x\left(u_{0}\right)$.

Let $\overline{K_{i}^{ \pm}}(p)$ and $K_{i}(p),(i=1,2)$ be the eigenvalues of $S_{p}^{ \pm}$and $A_{p}$. Since

$$
S_{p}^{ \pm}=-1_{T_{p} M} \pm A_{p}
$$

$S_{p}^{ \pm}$and $A_{p}$ have same eigenvectors and relations

$$
\overline{K_{i}^{ \pm}}(p)=-1 \pm K_{i}(p) .
$$

$\overline{K_{i}^{ \pm}}(p)$ and $K_{i}(p),(i=1,2)$ are called hyperbolic and de Sitter principal curvetures of $M$ at $p=x\left(u_{0}\right)$.

Let $\gamma(s)$ be a unit speed curve on $M$, with $p=\gamma\left(u_{1}(s), u_{2}(s)\right)$. We consider the hyperbolic curvature vector $k(s)=t^{\prime}(s)-\gamma(s)$ and the de Sitter normal curvature

$$
K_{n}^{ \pm}\left(s_{0}\right)=\left\langle k\left(s_{0}\right), L^{ \pm}\left(u_{1}\left(s_{0}\right), u_{2}\left(s_{0}\right)\right)\right\rangle=\left\langle t^{\prime}\left(s_{0}\right), L^{ \pm}\left(u_{1}\left(s_{0}\right), u_{2}\left(s_{0}\right)\right)\right\rangle+1
$$

of $p=\gamma\left(u_{1}\left(s_{0}\right), u_{2}\left(s_{0}\right)\right)$. The de Sitter normal curvature depends only on the point $p$ and the unit tangent vector of $M$ at $p$. Hyperbolic normal curvature of $\gamma(s)$ is defined to be

$$
\bar{K}_{n}^{ \pm}(s)=K_{n}^{ \pm}(s)-1
$$

The Hyperbolic Gauss curvature and mean curvature of $M$ at $p=x\left(u_{0}\right)$ is given by

$$
\overline{K_{h}^{ \pm}}\left(u_{0}\right)=\operatorname{det} S_{p}^{ \pm}=\overline{K_{1}^{ \pm}}(p) \overline{K_{2}^{ \pm}}(p)
$$

and

$$
H_{h}^{ \pm}\left(u_{0}\right)=\frac{1}{2} \operatorname{TraceS}_{p}^{ \pm}=\frac{\overline{K_{1}^{ \pm}}(p)+\overline{K_{2}^{ \pm}}(p)}{2}
$$

And also the extrinsic (de Sitter) Gauss curvature and mean curvature of $M$ at $p=x\left(u_{0}\right)$ is given by 


$$
K_{e}\left(u_{0}\right)=\operatorname{det} A p=K_{1}(p) K_{2}(p)
$$

and

$$
H_{d}\left(u_{0}\right)=\frac{1}{2} \operatorname{TraceA} p=\frac{K_{1}(p)+K_{2}(p)}{2}
$$

Let $x: M \rightarrow R_{1}^{4}$ be an immersion of a surface $M$ into $R_{1}^{4}$. We say that $x$ is timelike (resp. spacelike, lightlike) if the induced metric on $M$ via $x$ is Lorentzian (resp. Riemannian, degenerated). If $\langle x, x\rangle=1$, then $x$ is an immersion of $S_{1}^{3}$.

Let $x: M \rightarrow S_{1}^{3}$ be a spacelike immersion, and let $\xi$ be a unit normal vector field to $M$. If there exists spacelike direction $W$ such that timelike angle $\theta(\xi, U)$ is constant on $M$, then $M$ is called constant timelike angle surfaces with spacelike axis.

Let $x: M \rightarrow S_{1}^{3}$ be a spacelike immersion and let $\xi$ be a unit normal vector field to $M$. If there exists spacelike direction $W$ such that spacelike angle $\theta(\xi, U)$ is constant on $M$, then $M$ is called constant spacelike angle surfaces with spacelike axis.

From now on, the constant angle surface is proposed in de Sitter space $S_{1}^{3}$.

\section{TIMELIKE SURFACE WITH CONSTANT TIMELIKE ANGLE}

Let call $\chi(M)$ is tangent vector fields space over $M$. Let write $\overline{\bar{D}}, \bar{D}$ and $D$ are Levi-Civita connections of $R_{1}^{4}, S_{1}^{3}$ and $M$ respectively. For any $X, Y \in \chi(M)$, we have

$$
D_{X} Y=\left(\overline{\bar{D}}_{X} Y\right)^{T} D_{X} Y=\left(\overline{\bar{D}}_{X} Y\right)^{T}, \tilde{V}(X, Y)=\left(\overline{\bar{D}}_{X} Y\right)^{\perp}
$$

here $\tilde{V}$ is second fundamental form of $M$ over $R_{1}^{4}$ and

$$
\overline{\bar{D}}_{X} Y=\bar{D}_{X} Y-\langle X, Y\rangle x, \overline{\bar{D}}_{X} Y=D_{X} Y+\tilde{V}(X, Y)
$$

where the superscript $T$ and $\perp$ are the tangent and normal component of $\overline{\bar{D}}_{X} Y$. Equations in (3.1) are called the Gauss formula of $M$ on $S_{1}^{3}$. If $\xi$ is a normal vector field of M over $S_{1}^{3}$, then $A_{\xi}(X)$ and $B_{x}(X)$ Weingarten Endomorphism are defined by the tangent components of $-\overline{\bar{D}}_{X} \xi$ and $-\overline{\bar{D}}_{X} x$. So the Weingarten equations of the vector field $\xi$ and $x$ will be as follows

$$
\left\{\begin{array}{l}
A_{\xi}(X)=-\overline{\bar{D}}_{X} \xi-\left\langle\overline{\bar{D}}_{X} x, \xi\right\rangle x \\
B_{x}(X)=-\overline{\bar{D}}_{X} x+\left\langle\overline{\bar{D}}_{X} x, \xi\right\rangle \xi
\end{array}\right.
$$

It is clear that $A_{\xi}(X)$ and $B_{x}(X)$ operators for each $p \in M$ are both linear and self adjoint operators. That is 


$$
\left\langle A_{\xi}(X), Y\right\rangle=\left\langle X, A_{\xi}(Y)\right\rangle \text { and }\left\langle B_{x}(X), Y\right\rangle=\left\langle X, B_{x}(Y)\right\rangle \text {. }
$$

Let called eigenvalues $K_{i}(P)$ of $\left(A_{\xi}\right)_{p}$ over $S_{1}^{3}$ and eigenvalues $\tilde{K}_{i}(P)$ of $\left(B_{x}\right)_{p}$ over $R_{1}^{4}$ are principal curvetures and also, for any $X, Y \in \chi(M)$ we have

$$
\left\langle A_{\xi}(X), Y\right\rangle=\langle\widetilde{V}(X, Y), \xi\rangle,\left\langle B_{x}(X), Y\right\rangle=\langle\widetilde{V}(X, Y), x\rangle .
$$

Since $\tilde{V}(X, Y)$ is second fundamental form of $M$ over $I R_{1}^{4}$, so we can write $\tilde{V}(X, Y)$ as follows

$$
\widetilde{V}(X, Y)=\left\langle A_{\xi}(X), Y\right\rangle \xi+\left\langle B_{x}(X), Y\right\rangle x
$$

Let call $\left\{v_{1}, v_{2}\right\}$ is a base of TpM tangent plane and let us denote

$a_{i j}=\left\langle\widetilde{V}\left(v_{i}, v_{j}\right), \xi\right\rangle=\left\langle A_{\xi}\left(v_{i}\right), v_{j}\right\rangle$

$b_{i j}=\left\langle\widetilde{V}\left(v_{i}, v_{j}\right), x\right\rangle=\left\langle B_{x}\left(v_{i}\right), v_{j}\right\rangle$

So $\overline{\bar{D}}_{X} Y=D_{X} Y+\tilde{V}(X, Y)$. On the other hand for $\left\{v_{1}, v_{2}\right\}$ base, we get

$\overline{\bar{D}}_{v_{i}} v_{j}=D_{v_{i}} v_{j}-\left\langle A_{\xi}\left(v_{i}\right), v_{j}\right\rangle \xi-\left\langle v_{i}, v_{j}\right\rangle x$

If $\left\{v_{1}, v_{2}\right\}$ is orthonormal base, then we have from (3.1) and (3.2)

$\overline{\bar{D}}_{v_{i}} v_{j}=D_{v_{i}} v_{j}-a_{i j} \xi$

and also we get

$\overline{\bar{D}}_{v_{i}} \xi=-a_{i 1} v_{1}-a_{i 2} v_{2}$

$\overline{\bar{D}}_{v_{i}} x=b_{i 1} v_{1}-b_{i 2} v_{2}$

\subsection{Constant Timelike Angle Surfaces With Spacelike Axis}

Definition 1 Let $U \subset I R^{2}$ be open set and let $x: U \rightarrow S_{1}^{3}$ be an embedding where $M=x(U)$. Let $x: M \rightarrow S_{1}^{3}$ and $\xi$ is spacelike unit normal vector field on $M$, if there exist a constant spacelike vector $W$ which has a constant timelike angle with $\xi$, then $M$ is called constant timelike angle surface with spacelike axis. 
Since our surface is timelike, the orthogonal base of tangent space $T p M$ has a timelike tangent vector. Let $M$ be constant timelike angle surface with spacelike axis, and let $\xi$ and $W$ be unit normal and fixed axis of $M$. If $\theta$ is an timelike angle between spacelike vectors $\xi$ and $W$ then

$$
\langle\xi, W\rangle=-\cosh \theta
$$

If $\theta=0$, then $\xi=W$. From now on the rest of the paper, without loss of generality we assume that $\theta$. If $W^{T}$ is the projection of $W$ on the tangent plane of $M$, then we decompose $W$ as

$$
W=W^{T}+W^{N}
$$

So that we write

$$
W=W^{T}+\lambda_{1} \xi+\lambda_{2} x
$$

If we take inner product of both sides of this equality first with $\xi$, then with $x$

$$
\lambda_{1}=-\cosh \theta, \lambda_{2}=\langle W, x\rangle
$$

On the other hand since $W$ and $x$ are spacelike vector fields, then we can use define of spacelike and timelike angle between these vectors.

Theorem 2 If $\varphi$ is spacelike angle between spacelike vectors $W$ and $x$, then we can write from [11]

$$
W=\sqrt{\sin ^{2} \varphi-\cosh ^{2} \theta} \mid e_{1}-(\cosh \theta) \xi+(\cos \varphi) x
$$

and de Sitter projection $W_{d}$ of $W$ as follows

$$
W_{d}=\sqrt{\left|\sin ^{2} \varphi-\cosh ^{2} \theta\right|} e_{1}-(\cosh \theta) \xi
$$

Remark 1 Let $\varphi$ be timelike angle between spacelike vectors $W$ and $x$, then we can write for [11]

$$
\langle W, x\rangle=-\cosh \varphi
$$

or

$$
\lambda_{2}=-\cosh \phi .
$$

Therefore $W$ can be written as follows

$$
W=W^{T}-(\cosh \theta) \xi-(\cosh \varphi) x .
$$

On the other hand, since

$$
\left\|W^{T}\right\|^{2}=-\sinh ^{2} \theta-\cosh ^{2} \varphi
$$


then there is not any timelike angle between $W$ and $x$.

$$
\text { Let } e_{1}=\frac{W^{T}}{\left\|W^{T}\right\|} \text {, and let assume } e_{2} \text { be a unit vector field on } M \text { orthogonal to } e_{1} \text {. Then }
$$

we have an ortonormal basis $\left\{e_{1}, e_{2}, \xi, x\right\}$ in $R_{1}^{4}$ for each point of $M$. Since $W_{d}$ is constant vector field on $S_{1}^{3}$, we have

$$
\overline{\bar{D}}_{e_{2}} W_{d}=\bar{D}_{e_{2}} W_{d}=0
$$

hence we have

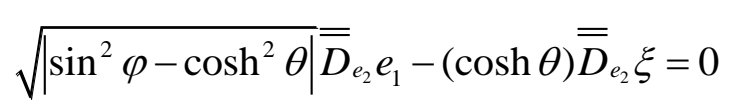

By (3.10) $\xi$, we obtain

$$
-\sqrt{\sin ^{2} \varphi-\cosh ^{2} \theta} \mid a_{21}=0
$$

Since $-\sqrt{\left|\sin ^{2} \varphi-\cosh ^{2} \theta\right|} \neq 0$, we conclude $a_{21}=a_{12}=0$. Using (3.7) in (3.10), we find

$$
\overline{\bar{D}}_{e_{2}} e_{1}=\frac{-\cosh \theta}{\sqrt{\left|\sin ^{2} \varphi-\cosh ^{2} \theta\right|}} a_{22} e_{2}
$$

Similarly, since $W_{d}$ is a constant vector field on $S_{1}^{3}$, then we have

$$
\bar{D}_{e_{1}} W_{d}=0 \text { and } \overline{\bar{D}}_{e_{1}} W_{d}=\sqrt{\left|\sin ^{2} \varphi-\cosh ^{2} \theta\right|} x
$$

By (3.9), we see that

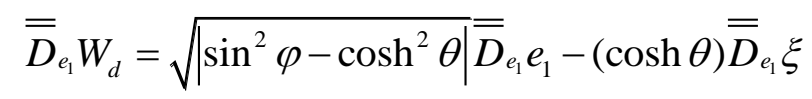

By (3.12) and (3.13), we conclude that

$$
\sqrt{\left|\sin ^{2} \varphi-\cosh ^{2} \theta\right|} \overline{\bar{D}}_{e_{1} e_{1}}-(\cosh \theta) \overline{\bar{D}}_{e_{1}} \xi=\sqrt{\left|\sin ^{2} \varphi-\cosh ^{2} \theta\right|} x
$$

By (3.14), then we get

$$
\sqrt{\left|\sin ^{2} \varphi-\cosh ^{2} \theta\right|}\left\langle\overline{\bar{D}}_{e_{1}} e_{1}, \xi\right\rangle=0
$$

or

$$
-\sqrt{\left|\sin ^{2} \varphi-\cosh ^{2} \theta\right|} a_{11}=0
$$


Since $-\sqrt{\left|\sin ^{2} \varphi-\cosh ^{2} \theta\right|} \neq 0$, we conclude $a_{11}=0$. Using (3.7) in (3.12), we obtain

$$
\overline{\bar{D}}_{e_{1}} e_{1}=x
$$

Hence, we have proved the following theorem.

Theorem 3 If $D$ is Levi-Civita connection for a constant timelike angle surface $M$ of $S_{1}^{3}$, then

$$
\begin{array}{ll}
D_{e_{1}} e_{1}=0 & D_{e_{2}} e_{1}=\frac{-\cosh \theta}{\sqrt{\left|\sin ^{2} \varphi-\cosh ^{2} \theta\right|}} a_{22} e_{2} \\
D_{e_{1}} e_{2}=0 & D_{e_{2}} e_{2}=\frac{-\cosh \theta}{\sqrt{\left|\sin ^{2} \varphi-\cosh ^{2} \theta\right|}} a_{22} e_{1} .
\end{array}
$$

Corollary 1 Let $M$ be a timelike surface with a constant timelike angle in $S_{1}^{3}$. Then, there exist local coordinates $u, v$ such that the metric on $M$ writes as $\langle\rangle:,=-d u^{2}+\beta^{2} d v^{2}$, where $\beta=\beta(u, v)$ is a smooth function on $M$, i.e. the coefficients of the first fundamental form are $E=-1, F=0, G=\beta^{2}$.

Let we find the $x=x(u, v)$ parametrization of the surface $M$ with respect to the metric $\langle\rangle:,=-d u^{2}+\beta^{2} d v^{2}$ on $M$. By Theorem-1, one can obtain the following corollary.

Corollary 2 There exist an equation system for a timelike surface with a constant timelike angle in $S_{1}^{3}$ which is

$$
\left\{\begin{array}{l}
x_{u u}=x \\
x_{u v}=\frac{\beta_{u}}{\beta} x_{v} \\
x_{v v}=\beta \beta_{u} x_{u}+\frac{\beta_{v}}{\beta} x_{v}-\beta^{2} a_{22} \xi-\beta^{2} x
\end{array}\right.
$$

Corollary 3 Let $\xi$ be unit normal vector of the a timelike surface with a constant timelike angle $M$. Then the equation below hold

$$
\left\{\begin{array}{l}
\xi_{u}=\overline{\bar{D}}_{x_{u}} \xi=0 \\
\xi_{v}=\overline{\bar{D}}_{x_{v}} \xi=-a_{22} x_{v}
\end{array}\right.
$$

Since $\xi_{u v}=\xi_{v u}$, we have $\overline{\bar{D}}_{x_{u}}\left(-a_{22} x_{v}\right)=0$. Using $a_{22}=0, \overline{\bar{D}}_{x_{u}} x_{v}=\overline{\bar{D}}_{x_{v}} x_{u}$ and Theorem 1, we obtain 
$\left(a_{22}\right)_{u}-\frac{\cosh \theta}{\sqrt{\left|\sin ^{2} \varphi-\cosh ^{2} \theta\right|}}\left(a_{22}\right)^{2}=0$

or

$\left(a_{22}\right)_{u}+\frac{\beta_{u}}{\beta}\left(a_{22}\right)=0$

Hence, we have

$\left(\beta a_{22}\right)_{u}=0$

By (3.20),we see that there exist a smooth function $\psi=\psi(v)$ depending on $v$ such that

$\beta a_{22}=\psi(v)$

Prposition 1 Let $x=x(u, v)$ be parametrization of a timelike surface with a constant timelike angle in $S_{1}^{3}$. If $a_{22}=0$ on $M$, then the $x$ describes an flat plane of de Sitter space $S_{1}^{3}$.

From now on, we are assume that $a_{22} \neq 0$. By solving equation (3.16), we obtain a function $\alpha=\alpha(v)$ such that

$$
a_{22}=\frac{-\sqrt{\sin ^{2} \varphi-\cosh ^{2} \theta}}{u \cosh \theta+\alpha(v)}, \alpha(v)=\sqrt{\sin ^{2} \varphi-\cosh ^{2} \theta} \bar{\alpha}(v) .
$$

Therefore by (3.21), we obtain

$$
\beta(u, v)=\frac{-\psi(v)}{\sqrt{\sin ^{2} \varphi-\cosh ^{2} \theta}}(u \cosh \theta+\alpha(v)) .
$$

If we choose $\psi(v)=-v \sqrt{\sin ^{2} \varphi-\cosh ^{2} \theta}$ and $\alpha(v)=\ln v$, then we have the following theorem.

Theorem 4 If $M$ is satisfy (3.22), then there exist local coordinates $u, v$ on $M$ with having the parametrization

$x_{i}(u, v)=\frac{-d_{1 i}(v)}{2 \cosh \theta(u \cosh \theta+\ln v)^{2}}+d_{2 i}(v), i=1,2,3,4$

Proof From (3.22), the proof is clear. 


\section{REFERENCES}

[1]. R. Lopez, M.I. Munteanu, Constant angle surfaces in Minkowski space, Bulletin of the Belgian Math. So. Simon Stevin, Vo.18 (2011) 2, (271-286).

[2]. S.Izumıya, K.Saj1, M.Takahash1, Horospherical flat surfaces in Hyperbolic 3-space, J.Math.Soc.Japan, Vol.87 (2010), (789-849).

[3]. S.Izumıya, D.Pe1, M.D.C.R. Fuster, The horospherical geometry of surfaces in hyperbolic 4spaces, Israel Journal of Mathematics, Vol.154 (2006), (361-379).

[4]. C.Thas, A gauss map on hypersurfaces of submanifolds in Euclidean spaces, J.Korean Math.Soc., Vol.16, No.1, (1979).

[5]. S.Izumıya, D.Pe1, T.Sano, Singularities of hyperbolic gauss map, London Math.Soc. Vol.3 (2003), (485-512).

[6]. M.I.Munteanu, A.I.Nistor, A new approach on constant angle surfaces in $E^{3}$, Turk T.Math. Vol.33 (2009), (169-178).

[7]. C.Takızawa, K.Tsukada , Horocyclic surfaces in hyperbolic 3-space, Kyushu J.Math. Vol.63 (2009), (269-284).

[8]. S.Izumiya, M.D.C.R. Fuster, The horospherical Gauss-Bonnet type theorem in hyperbolic space, J.Math.Soc.Japan, Vol.58 (2006), (965-984).

[9]. B. O'Neill, Semi-Riemannıan Geometry with applications to relativity, Academic Press, New York, 1983.

[10].W.Fenchel, Elementary Geometry in Hyperbolic Space, Walter de Gruyter, New York, 1989.

[11].J.G.Ratcliffe, Foundations of Hyperbolic Manifolds, Springer 1948.

[12].P.Cermelli, A.J. Di Scala, Constant angle surfaces in liquid crystals, Phylos. Magazine,Vol.87 (2007),(1871-1888).

[13].A.J. Di Scala, G. Ruiz-Hernandez, Helix submanifolds of Euclidean space, Monatsh. Math. DOI 10.1007 / s00605-008-0031-9.

[14].G.Ruiz-Hernandez, Helix, shadow boundary and minimal submanifolds, Illinois J. Math. Vol.52 (2008),(1385-1397).

[15].F. Dillen, J. Fastenakels, J. Van der Veken, L. Vrancken, Constant angle surfaces in $S^{2} \times R$, Monaths. Math., Vol.152 (2007), (89-96).

[16].F. Dillen and M. I. Munteanu, Constant angle surfaces in $H^{2} \times R$, Bull. Braz. Math. soc. , Vol.40 (2009), (85-97).

[17].J. Fastenakels, M. I: Munteanu , J. Van der Veken, Constant angle surfaces in the Heisenberg group, Acta Math. Sinica (English Series) Vol.27 (2011), (747-756).

[18].R.Lopez, Differantial Geometry of Curves and Surfaces in Lorentz-Minkowski space, arXiv: 0810.3351 (2008).

[19].Mert.T, Karliğa.B, Constant Angle Spacelike Surface in Hyperbolic Space $H^{3}$, J. Adv. Res. Appl. Math., 7(2015), no 2, (89-102).

[20].Mert.T, Karliğa.B, Constant Angle Spacelike Surface in de Sitter Space $S_{1}^{3}$, Boletim da Sociedade Paranaense de Mathematica (Accepted).

[21].Mert.T, Karliğa.B, On The Timelike Surface with Constant Angle in Hyperbolic Space $H^{3}$, CBU Journal of Science (Accepted).

[22].M. Kasedou, Spacelike submanifolds in de sitter space, demonstratio Mathematica, Vol.XLIII (2010), 2. 